\title{
Comparison of OSTA, FRAX and BMI for Predicting Postmenopausal Osteoporosis in a Han Population in Beijing: A Cross Sectional Study
}

This article was published in the following Dove Press journal: Clinical Interventions in Aging

\author{
Zihan Fan \\ Xiaoyu Li \\ Xiaodong Zhang (D) \\ Yong Yang \\ Qi Fei (D) \\ Ai Guo \\ Department of Orthopedics, Beijing \\ Friendship Hospital, Capital Medical \\ University, Beijing, People's Republic of \\ China
}

Purpose: To validate the efficacies of three screening tools including the Osteoporosis SelfAssessment Tool for Asians (OSTA), Fracture Risk Assessment Tool (FRAX) without bone mineral density (BMD), and body mass index (BMI) for predicting postmenopausal osteoporosis (OP) and to define the ideal thresholds for avoidance of dual-energy X-ray absorptiometry (DXA) scanning in a Han Chinese population in Beijing.

Patients and Methods: A total of 2055 community-dwelling Han Beijing postmenopausal females aged $\geq 45$ years were enrolled in this study. All participants completed a questionnaire, and BMD was measured by DXA. OP was defined by a T-score at least -2.5 SD less than that of average young adults in different diagnostic criteria [lumbar spine, femoral neck, total hip, worst hip, WHO]. The abilities of the OSTA, FRAX, and BMI to predict OP were analyzed by receiver operating characteristic (ROC) curves. Sensitivity, specificity, and area under the ROC curves (AUC) were calculated. Ideal thresholds for identifying OP were proposed.

Results: The prevalence of OP ranged from $8.1 \%$ to $28.4 \%$ according to different diagnostic criteria. The AUC range for the OSTA (0.758-0.849) was similar to the FRAX (0.7280.855), which revealed that both tools predicted OP reliably. The AUC range for BMI was 0.643-0.682, suggesting limited predictive value. According to WHO criteria, the AUC values for the FRAX for hip fracture risk (FRAX-HF) and for the OSTA were 0.796 and 0.798 , with corresponding sensitivities of $74.79 \%$ and $69.64 \%$ and specificities of $70.45 \%$ and $75.07 \%$, respectively. At defined thresholds, the FRAX-HF and OSTA allowed avoidance of DXA in $42.4-37.6 \%$ of participants, at a cost of missing only $7.2-8.6 \%$ of individuals with OP.

Conclusion: The OSTA and FRAX-HF may be reliable and effective tools for identifying postmenopausal OP in the Han Beijing population without BMD.

Keywords: postmenopausal osteoporosis, OSTA, FRAX, BMI

\section{Introduction}

Postmenopausal osteoporosis (OP) is the most common skeletal metabolic disorder that affects postmenopausal women and involves microarchitectural deterioration and bone mineral density (BMD) reduction caused by menopause-related estrogen deprivation and increasing age. ${ }^{1}$ Postmenopausal women with OP have a high susceptibility to fragility fractures. ${ }^{2}$ Due to the progressive aging trend of the population, the morbidity and mortality of OP and associated fractures grows year by year, ${ }^{3}$ creating an ever-increasing burden for clinical resources and economic expenses. ${ }^{4,5}$ In the USA, the annual cost for OP-related fractures in 2005 was
Correspondence: Qi Fei; Ai Guo Department of Orthopedics, Beijing Friendship Hospital, Capital Medical University, No. 95, Yong'an Road, Xicheng District, Beijing 100050, People's Republic of China

Tel +861063138353

Fax +86108391 1029

Email spinefei@I26.com;

guoaiortho@I26.com 
17 billion dollars, with $71 \%$ of fractures occurring in women and the costs for treatment of such fractures being $75 \%$ higher than those in men, and a further $50 \%$ increase in cost to 25.3 billion dollars are projected by $2025 .^{6}$ The situation of OP in China is equally serious, as it has been predicted that the osteoporotic population will expand sharply from 83.9 million in 1997 to 212 million by $2050 .^{7}$ Osteoporotic fractures require the longest perhospitalization length of stay and highest cost among all fractures $^{8}$ for Chinese patients. By 2010, an estimated 2.33 million OP-related fractures had occurred with a treatment cost of 9.45 billion dollars. ${ }^{9}$ To make matters worse, the annual number of cases and costs in OP-related fractures are estimated to be double by 2035 , and will increase to 5.99 million fractures costing 25.43 billion dollars by 2050 in China. ${ }^{9}$ Considering the high morbidity and social costs of OP and fragility fractures, efforts to develop convenient and reliable screening tools for postmenopausal women are crucial to reduce the clinical and economic burdens in the aging society.

BMD measured by dual-energy X-ray absorptiometry (DXA) is currently considered to be the gold standard for the diagnosis of OP. ${ }^{1}$ According to the World Health Organization (WHO) criteria, OP is defined by a low BMD more than 2.5 standard deviations (SD) below the mean BMD for young healthy adults at any site of the lumbar spine (L1-L4), femoral neck, or total hip. ${ }^{10}$ According to the National Osteoporosis Foundation (NOF) guidelines, treatments should be initiated when DXA shows a T-scores at least -2.5 SDs at any site. ${ }^{11}$ Nevertheless, screening for OP by DXA is not feasible due to its high cost, poor portability, and patient exposure to ionizing radiation. It's not convenient for communitydwelling elderly people to have access to the expensive DXA devices at community health service centers, particularly for those who live in suburban or rural areas. The prevalence rates of asymptomatic OP (22.66\%) and osteopenia $(47.36 \%)$ among Chinese postmenopausal women were both high in a previous study. ${ }^{12}$ Hence, identifying and prescreening OP is important to prioritize individuals at risk of OP for DXA scanning. Results obtained by using credible risk assessment tools could help clinicians decide whether further DXA examination is needed for patients.

The Fracture Risk Assessment Tool (FRAX) developed by the University of Sheffield, as a computer-based algorithm, is designed to predict the 10-year probability of hip fracture (HF) and major osteoporotic fracture (MOF) according to an individual's clinical risk factors with or without BMD. ${ }^{13}$ FRAX without BMD is not only well established for evaluating the risk of osteoporotic fractures, but also as an effective tool for identifying OP. ${ }^{14,15}$ Although FRAX cannot replace BMD measurement in guiding treatment, it has been reported that FRAX provides useful information to guide clinical decisions regarding anti-OP medication use. ${ }^{16}$ In the recent UK National Osteoporosis Guideline Group (NOGG) guidance, treatment is recommended in elderly osteoporotic patients with a FRAX-MOF of at least $20 \%$ or FRAX-HF of at least $5 \% .{ }^{17}$ Thus, the unnecessary BMD may be able to be omitted in the individuals at high risk based on FRAX. However, the setting of intervention thresholds must still be country-specific. ${ }^{15}$ Expanding on a few studies on the ability of FRAX to estimate the risk of OP in Chinese postmenopausal women, the present study was conducted to define the screening threshold of FRAX without BMD for OP.

The Osteoporosis Self-Assessment Screening Tool for Asians (OSTA) was established in 2001 based on an analysis of 860 community dwelling postmenopausal women enrolled from various Asian regions. ${ }^{18}$ Although only two factors (age and weight) are considered, the OSTA still proved to be a convenient and valid screening tool for predicting the risk of OP in postmenopausal women. ${ }^{19}$ Notably, poor results were reported for validation of the OSTA's efficacy in predicting postmenopausal OP in a Chinese cohort (not Beijing). ${ }^{20,21}$ The efficacy and cutoff value for the OSTA may vary among diverse populations and diagnostic criteria. ${ }^{22}$ Therefore, it is necessary to revalidate the tool according to the local demographic profile, before OSTA can be applied clinically for OP screening.

Body mass index (BMI) is well established as an international standard for evaluating obesity and health in individuals. In addition, BMI has also shown a positive association with BMD. ${ }^{23}$ Women less than 60 years old with low BMI $\left(<20 \mathrm{~kg} / \mathrm{m}^{2}\right)$ have been recommended to undergo DXA based on the results of a previous study in Europe,${ }^{24}$ but the validity of BMI as an OP screening tool remains uncertain. Because a certain correlation between BMI and BMD was verified in postmenopausal women, ${ }^{25}$ we proposed to estimate the validity of BMI as a screening method for OP in Chinese postmenopausal women, specifically in comparison with the OSTA and FRAX without BMD.

The purpose of this study was to compare the efficacies of the OSTA, FRAX without BMD, and BMI for 
predicting postmenopausal OP and to confirm the ideal thresholds to reduce unnecessary use of BMD among community dwelling postmenopausal women in Beijing.

\section{Patients and Methods}

The study was approved by the ethics committee of Beijing Friendship Hospital, Capital Medical University (BJFH-EC/2013-013). We confirm that this study was conducted in accordance with the Declaration of Helsinki. All participants provided signed informed consent for enrollment in this study. A flow diagram of the study is shown in Figure 1.

\section{Study Design}

The study population for this cross-sectional study included healthy postmenopausal women (age $\geq 45$ years) consecutively enrolled from communities near Beijing Friendship hospital in the Xicheng District of Beijing from January 2013 to October 2017. The main inclusion and exclusion criteria are listed in Table 1 . None of the recruited participants had ever been diagnosed with OP prior to the study.

\section{BMD Measurements and Data Collection via Questionnaire}

All participants were healthy Beijing community-dwelling postmenopausal women who came to the OP clinic in Beijing Friendship hospital for DXA BMD measurements at the hip and spine. The participants were required to complete a questionnaire with a trained interviewer to provide information regarding demographic variables and clinical risk factors for OP using a structured table. The collected data included age, height, weight, history of previous fracture, current smoking, alcoholic drinks per day, history of parent hip fracture, and so on. Height was measured using a stadiometer (Mahr GmbH, Gottingen, Germany), and weight was measured with an electronic balance (Tanita, Tokyo, Japan). The database was established and checked by three researchers (Zihan Fan,

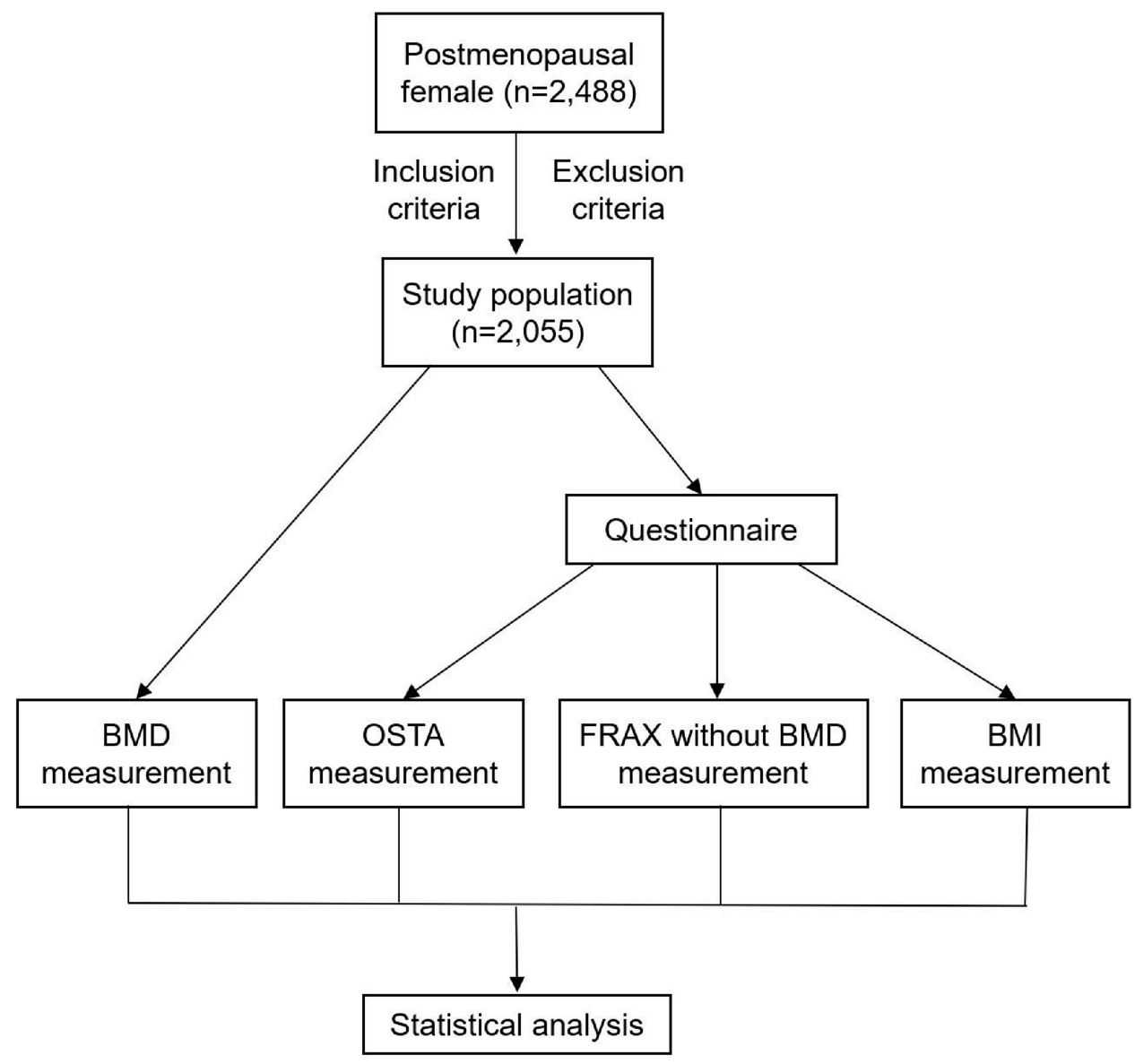

Figure I The flow diagram of the study.

Abbreviations: BMD, bone mineral density; OSTA, Osteoporosis Self-Assessment Tool for Asians; FRAX, Fracture Risk Assessment Tool; BMI, body mass index. 
Table I Inclusion and Exclusion Criteria for This Study

\begin{tabular}{|c|c|}
\hline Inclusion Criteria & Exclusion Criteria \\
\hline $\begin{array}{l}\text { Han Chinese nationality. } \\
\text { Menses stops for at least } 12 \\
\text { months. } \\
\text { Postmenopausal women } \\
\text { (age } \geq 45 \text { years). } \\
\text { Residency in Beijing for } \geq 20 \\
\text { years. } \\
\text { Ability to read and provide } \\
\text { informed consent. }\end{array}$ & $\begin{array}{l}\text { Rheumatoid arthritis } \\
\text { History of glucocorticoid use. } \\
\text { History of thyroid supplements, } \\
\text { antidepressant treatment. } \\
\text { History of metabolic bone disease (eg, } \\
\text { type I diabetes, hyperparathyroidism } \\
\text { or hypoparathyroidism, Paget's } \\
\text { disease, osteomalacia, renal } \\
\text { osteodystrophy, osteogenesis } \\
\text { imperfecta). } \\
\text { History of organ transplantation. } \\
\text { History of taking anti-OP medications. } \\
\text { History of malignant tumor. } \\
\text { Operation of lumbar spine or bilateral } \\
\text { hips. } \\
\text { Significant renal or hepatic } \\
\text { impairment. }\end{array}$ \\
\hline
\end{tabular}

Xiaoyu Li and Xiaodong Zhang) to guarantee the quality of data and then rechecked by the senior researchers (Yong Yang, Qi Fei and Ai Guo). The BMDs of the femur (the femoral neck and total hip) and lumbar spine (L1-L4) were assessed by DXA (Discovery Wi, QDR Series; Hologic, Waltham, MA, USA). To ensure conformity of measurements, DXA was operated and calibrated by the same qualified and well-trained technician. All data such as height, weight and BMD were measured using the same equipment throughout the study. The in vivo short-term reproducibility values were all $<1 \%$ for all measurements of the femoral neck, total hip and lumbar spine BMDs. The mean values of young Chinese women which had been set as the reference population were used to calculate the T-scores: femoral neck $0.803 \pm 0.101 \mathrm{~g} / \mathrm{cm}^{2}$, and total hip BMD 0.864 $\pm 0.113 \mathrm{~g} / \mathrm{cm}^{2}$, L1-L4 BMD 0.967 \pm 0.109 $\mathrm{g} / \mathrm{cm}^{2}$. OP was defined by a T-score of -2.5 or lower at different skeletal sites, ${ }^{26}$ according to various criteria including the lumbar spine (L1-L4), femoral neck, total hip, worst hip (femoral neck or total hip) and the WHO (any site).

\section{OSTA Score}

The OSTA score was deduced and based on weight in kilograms and age in years after multiple variable regression analysis in $2001^{18}$ using the algorithm below:

$$
\text { OSTA }=[\text { body weight }(\mathrm{kg})-\operatorname{age}(\text { years })] \times 0.2
$$

The decimal digits were dropped in order to truncate the results to the integer. For example, a 66-year-old woman weighing $50 \mathrm{~kg}$ would have an OSTA index of -3 .

\section{FRAX Score}

FRAX was well known as a computer-based screening tool developed by Kanis et $\mathrm{al}^{13}$ which calculated the 10 year probability of a major osteoporotic fracture (FRAXMOF) and a hip fracture (FRAX-HF). The calculation of fracture risks was based on the individual's information including age, gender, weight, height, and several wellvalidated dichotomized risk factors with or without BMD. Because this study focused on evaluating the efficacy of FRAX as a tool in identifying OP for the Chinese postmenopausal female population, the data were all collected without BMD measurement.

\section{BMI}

BMI was calculated as weight divided by the square of height $\left(\mathrm{kg} / \mathrm{m}^{2}\right)$ using the following formula:

$$
B M I=\frac{\text { Body weight }(K g)}{\operatorname{Body} \text { height }(m)^{2}}
$$

\section{Statistical Analysis}

The performance of the different tools (OSTA, FRAX without $\mathrm{BMD}$, and $\mathrm{BMI}$ ) in predicting $\mathrm{OP}$ at the lumbar spine, total hip, femoral neck, worst hip (femoral neck or total hip), and any site (WHO criteria) was evaluated and compared. OP was defined as mentioned above. The receiver operating characteristic (ROC) curves were plotted, and the area under the ROC curve (AUC) and its 95\% confidence interval (CI) were computed and compared for each test using SPSS Version 21 (IBM Corporation, Armonk, NY, USA) and MedCalc version 11.5.0.0 (MedCalc Software, Ostend, Belgium). The predictive efficacies of the above tools were estimated according to the AUC values as follows: $\mathrm{AUC}=1$, perfectly predictive; $0.9 \leq \mathrm{AUC}<1$, highly predictive; $0.7 \leq \mathrm{AUC}<0.9$, moderately predictive; $0.5 \leq \mathrm{AUC}<0.7$, less predictive; and AUC $<0.5$, non-predictive. ${ }^{27}$ AUC values $>0.75$ were generally considered to indicate good performance of the diagnostic tool. ${ }^{28}$ The independent-samples $t$-test and one-way analysis of variance were applied for single-factor analysis. The data were initially collated in a Microsoft Excel (Microsoft Corporation, Redmond, WA) spreadsheet, and the analysis was performed using SPSS Version 21 (IBM Corporation). 
We calculated the ideal thresholds based on the results for AUCs in order to maximize the diagnostic benefit and minimize missed diagnosis. Then we calculated the sensitivity, specificity, and positive and negative predictive values for each threshold, and the results were also transformed into number of cases for which BMD measurement was avoided per 100 individuals (candidates would be screened) and the percentage of participants with OP who would be missed. ${ }^{26}$

\section{Results}

A total of 2488 postmenopausal healthy women aged 45 or older were recruited for participation in this study. According to the inclusion and exclusion criteria, 2055 individuals were eligible for analysis. The characteristics of the participants are shown in Table 2.

According to the WHO, lumbar spine, worst hip, femoral neck, and total hip criteria, the prevalence rates of OP in this study were $28.4 \%, 19.9 \%, 18.1 \%, 17.2 \%$, and $8.1 \%$, respectively.

\section{ROC Curve Outcomes}

The ROC curves for each tool according to the different criteria were analyzed, and the corresponding thresholds were estimated. A summary of the cutoff values and AUCs is presented in Table 3. The AUC values of the tools for predicting $\mathrm{OP}$ according to the five diagnostic criteria (WHO, lumbar spine, worst hip, femoral neck, and total hip criteria) ranged from 0.758 to 0.849 (OSTA), 0.750 to 0.855 (FRAX-HF), 0.728 to 0.829 (FRAX-MOF), and 0.643 to 0.682 (BMI). With the WHO criteria, the FRAX-HF and OSTA had the highest AUC values (0.796 and 0.798), and there was no significant difference between them $(p=0.78)$. Thus, the FRAX-HF and OSTA yielded the best predictive value among these tools. The ROC curves and AUCs for the WHO criteria are presented in Figure 2.

\section{Lower Threshold Results}

In this study, the AUCs for the three tools revealed the best results in the total hip criterion. However, the prevalence rate with this criterion was only $8.1 \%$, which was much lower than the rate with the other criteria and did not represent the real prevalence of OP. The low prevalence may increase the frequency of missed diagnosis with the screening tools; hence, we set the cutoff value in the WHO criterion as the optimal threshold. The ROC curves, AUCs and cutoff values for the WHO criteria are presented in Figure 2. The lower
Table 2 The Characteristics of Participants $(n=2055)$

\begin{tabular}{|c|c|c|}
\hline Characteristics & Value & Range \\
\hline $\begin{array}{l}\text { Age (year) } \\
\text { Height }(\mathrm{cm}) \\
\text { Weight }(\mathrm{kg}) \\
\text { Body mass index }\left(\mathrm{kg} / \mathrm{m}^{2}\right)\end{array}$ & $\begin{array}{l}62.06 \pm 9.12 \\
158.63 \pm 5.19 \\
60.58 \pm 9.34 \\
24.06 \pm 3.43\end{array}$ & $\begin{array}{l}45-90 \\
140-178 \\
30-106 \\
|2.82-39.4|\end{array}$ \\
\hline $\begin{array}{l}\text { Bone mineral density }\left(\mathrm{g} / \mathrm{cm}^{2}\right)^{\mathrm{a}} \\
\text { Lumbar Spine } \\
\text { Femoral neck } \\
\text { Total hip }\end{array}$ & $\begin{array}{l}0.832 \pm 0.152 \\
0.677 \pm 0.128 \\
0.778 \pm 0.139\end{array}$ & $\begin{array}{l}0.370-1.534 \\
0.259-1.160 \\
0.268-1.308\end{array}$ \\
\hline $\begin{array}{l}\text { Lumbar Spine, n (\%) } \\
\text { Normal } \\
\text { Osteopenia } \\
\text { Osteoporosis }\end{array}$ & $\begin{array}{l}855(41.6 \%) \\
791(38.5 \%) \\
409 \text { (19.9\%) }\end{array}$ & \\
\hline $\begin{array}{l}\text { Femoral neck, n (\%) } \\
\text { Normal } \\
\text { Osteopenia } \\
\text { Osteoporosis }\end{array}$ & $\begin{array}{l}654(31.8 \%) \\
1048(51.0 \%) \\
353(17.2 \%)\end{array}$ & \\
\hline $\begin{array}{l}\text { Total hip, n (\%) } \\
\text { Normal } \\
\text { Osteopenia } \\
\text { Osteoporosis }\end{array}$ & $\begin{array}{l}1203(58.5 \%) \\
685(33.3 \%) \\
167(8.1 \%)\end{array}$ & \\
\hline $\begin{array}{l}\text { Worst hip, }{ }^{\mathrm{b}} \mathrm{n}(\%) \\
\text { Normal } \\
\text { Osteopenia } \\
\text { Osteoporosis }\end{array}$ & $\begin{array}{l}628(30.5 \%) \\
1056(51.4 \%) \\
371(18.1 \%)\end{array}$ & \\
\hline $\begin{array}{l}\text { Worst any site, }^{c} \mathrm{n}(\%) \\
\text { Normal } \\
\text { Osteopenia } \\
\text { Osteoporosis } \\
\text { Alcohol } \geq 30 \mathrm{~g} / \mathrm{d}^{\mathrm{d}} \\
\text { Current smoking } \\
\text { Previous fracture }^{\mathrm{e}} \\
\text { Parent fractured hip }^{\mathrm{f}}\end{array}$ & $\begin{array}{l}469(22.8 \%) \\
1003(48.8 \%) \\
583(28.4 \%) \\
31(1.5 \%) \\
55(2.7 \%) \\
550(26.8 \%) \\
242(11.8 \%)\end{array}$ & \\
\hline
\end{tabular}

Notes: ${ }^{\mathrm{B} M M D}$ T-scores classified according to WHO criteria: osteoporosis $(\leq-2.5)$, osteopenia $(-1.0$ to -2.5$)$, and normal ( $\geq-1.0)$. ${ }^{b}$ Worst hip criteria, OP was defined by a BMD T-score of $\leq-2.5 \mathrm{SD}$ at femoral neck or total hip sites. ${ }^{\mathrm{C}} \mathrm{WHO}$ criteria, OP was defined by a BMD T-score of $\leq-2.5 \mathrm{SD}$ at any site of LI-L4, femoral neck, or total hip sites. ${ }^{\mathrm{d}}$ Taking $30 \mathrm{~g}$ or more of alcohol daily (equivalent to three units of alcohol in the present study). ${ }^{\mathrm{e}} \mathrm{Fractures}$ after the age of 45 years with or without low-energy trauma history. ${ }^{f}$ History of hip fracture in the patient's parents; subjects answered either yes or no.

Abbreviations: $\mathrm{BMD}$, bone mineral density; OP, osteoporosis.

thresholds were set at 1\% for FRAX-HF, 4.6\% for FRAXMOF, -1 for OSTA, and 22.67 for BMI.

The performances of the different tools at these thresholds are summarized in Table 4. With the WHO criteria, the FRAX-HF and OSTA provided the highest predictive efficacy according to the observed AUCs, with a sensitivity of $74.8-69.6 \%$, test saved rate of $42.4-37.6 \%$, and percentage of missed OP cases of $7.2-8.6 \%$. 
Table 3 Cutoff Value and AUC for Prediction of OP in Different Criteria

\begin{tabular}{|l|l|l|l|l|l|l|l|l|l|l|l|}
\hline Screening & \multicolumn{2}{l}{ Lumbar Spine } & \multicolumn{2}{l|}{ Total Hip } & \multicolumn{2}{l|}{ Femoral Neck } & \multicolumn{2}{l|}{ Worst Hip } & \multicolumn{2}{l|}{ WHO } \\
\hline Tool & Cutoff & AUC & Cutoff & AUC & Cutoff & AUC & Cutoff & AUC & Cutoff & AUC \\
\hline BMI & 23.19 & 0.667 & 22.66 & 0.682 & 22.64 & 0.647 & 22.64 & 0.643 & 22.67 & 0.654 \\
OSTA & -1 & 0.758 & -2 & 0.849 & -1 & 0.823 & -1 & 0.824 & -1 & 0.798 \\
FRAX-MOF & 4.6 & 0.728 & 6.8 & 0.829 & 4.4 & 0.794 & 4.5 & 0.798 & 4.6 & 0.768 \\
FRAX-HF & $\mathrm{I}$ & 0.750 & 1.9 & 0.855 & 1.3 & 0.825 & 1.3 & 0.828 & 1 & 0.796 \\
\hline
\end{tabular}

Abbreviations: AUC, area under the receiver operating characteristic curves; BMI, body mass index; OSTA, Osteoporosis Self-Assessment Tool for Asians; FRAX, Fracture Risk Assessment Tool; MOF, major osteoporotic fractures; HF, hip fractures; WHO, World Health Organization.

\section{Discussion}

This study compared the performances of the OSTA, FRAX without BMD, and BMI as prediction tools for postmenopausal OP in community-dwelling Han Beijing women aged $\geq 45$ years and attempted to define the optimal thresholds beyond which the unnecessary BMD testing for OP screening could be avoided. A recent systematic review reported that the prevalence of OP in Chinese women was $25.9 \%$, which is much higher than that in men $(5.30 \%){ }^{29}$ In the current study, a total of 2055 participants were finally eligible out of 2488 individuals, and the prevalence of OP ranged from $8.1 \%$ to $28.4 \%$ according to the different criteria. With the WHO criteria, the prevalence rates of osteopenia and osteoporosis among Han Beijing postmenopausal women were $48.8 \%$ and $28.4 \%$, respectively, which was basically consistent with previously reported rates. ${ }^{12,29}$ The high prevalence of OP emphasizes the need for a reliable and convenient screening tool to identify Chinese postmenopausal women at risk, because most OP patients are asymptomatic, making the diagnosis easy to be missed.

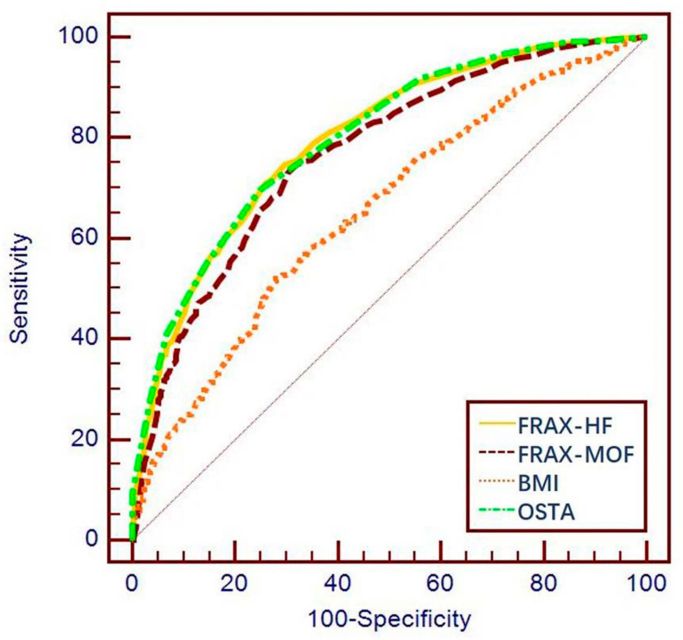

\begin{tabular}{llllllllll}
\hline & AUC(95\% Cl) & $\mathbf{Z}$ & p-value & Cutoff & Sensitivity(\%) & Specificity(\%) & Youden's & +LR & -LR \\
\hline OSTA & $0.798(0.780-0.815)$ & 28.64 & $<0.0001$ & -1.0 & 69.64 & 75.07 & 44.71 & 2.79 & 0.40 \\
FRAX-HF & $0.796(0.778-0.813)$ & 27.73 & $<0.0001$ & 1.0 & 74.79 & 70.45 & 45.24 & 2.53 & 0.36 \\
FRAX-MOF & $0.768(0.749-0.786)$ & 23.60 & $<0.0001$ & 4.6 & 73.07 & 69.63 & 42.70 & 2.41 & 0.39 \\
BMI & $0.654(0.633-0.675)$ & 11.52 & $<0.0001$ & 22.7 & 51.97 & 71.94 & 23.91 & 1.85 & 0.67 \\
\hline
\end{tabular}

Figure 2 Comparison of different AUCs (OSTA, FRAX-HF, FRAX-MOF and BMI for identifying OP), Youden's index, likelihood ratio, sensitivity and specificity values according to the WHO criteria.

Abbreviations: AUCs, areas under the receiver-operating characteristic curves; BMI, body mass index; FRAX, Fracture Risk Assessment Tool; HF, hip fractures; +LR, positive likelihood ratio; -LR, negative likelihood ratio; MOF, major osteoporotic fractures; OSTA, Osteoporosis Self-Assessment Tool for Asians. 
Table 4 Test Performance in Predicting OP at Defined Low-Risk Thresholds

\begin{tabular}{|c|c|c|c|c|}
\hline \multirow[t]{2}{*}{ Test Performance } & \multicolumn{2}{|c|}{$\begin{array}{l}\text { Ten-Year Fracture } \\
\text { Risk as Predicted } \\
\text { by FRAX without } \\
\text { BMD }\end{array}$} & \multirow[t]{2}{*}{$\begin{array}{l}\text { OSTA, } \\
\%\end{array}$} & \multirow[t]{2}{*}{$\begin{array}{l}\text { BMI, } \\
\%\end{array}$} \\
\hline & HF, \% & $\begin{array}{l}\text { MOF, } \\
\%\end{array}$ & & \\
\hline \multicolumn{5}{|l|}{ Lumbar spine $B M D \leq-2.5$} \\
\hline Sensitivity & 72.6 & 71.4 & 67.5 & 55.7 \\
\hline Specificity & 65.1 & 64.7 & 69.8 & 70.4 \\
\hline Positive predictive value & 34.1 & 33.4 & 35.7 & 31.8 \\
\hline Negative predictive value & 90.5 & 90.1 & 89.6 & 86.5 \\
\hline Tests saved ${ }^{\mathrm{a}}$ & 42.4 & 42.5 & 37.6 & 34.8 \\
\hline OP missed ${ }^{b}$ & 5.5 & 5.7 & 6.5 & 8.8 \\
\hline Cut-off value & 1.0 & 4.6 & -1.0 & 23.2 \\
\hline \multicolumn{5}{|l|}{ Femoral neck BMD $\leq-2.5$} \\
\hline Sensitivity & 84.1 & 80.7 & 78.5 & 53.3 \\
\hline Specificity & 66.3 & 65.5 & 70.9 & 69.0 \\
\hline Positive predictive value & 34.1 & 32.6 & 35.8 & 26.3 \\
\hline Negative predictive value & 95.3 & 94.2 & 94.1 & 87.7 \\
\hline Tests saved & 42.4 & 42.5 & 37.6 & 34.8 \\
\hline OP missed & 2.7 & 3.3 & 3.7 & 8.0 \\
\hline Cut-off value & 1.3 & 4.4 & -1.0 & 22.6 \\
\hline \multicolumn{5}{|l|}{ Total hip BMD $\leq-2.5$} \\
\hline Sensitivity & 89.8 & 86.8 & 85.6 & 61.7 \\
\hline Specificity & 61.8 & 61.4 & 66.6 & 67.5 \\
\hline Positive predictive value & 17.2 & 16.6 & 18.5 & 14.4 \\
\hline Negative predictive value & 98.6 & 98.1 & 98.1 & 95.2 \\
\hline Tests saved & 42.4 & 42.5 & 37.6 & 34.8 \\
\hline OP missed & 0.8 & 1.1 & 1.2 & 3.1 \\
\hline Cut-off value & 1.9 & 6.8 & -2.0 & 22.7 \\
\hline \multicolumn{5}{|l|}{ Worst hip BMD $\leq-2.5$} \\
\hline Sensitivity & 84.1 & 81.1 & 78.4 & 52.6 \\
\hline Specificity & 66.8 & 66.0 & 71.4 & 69.1 \\
\hline Positive predictive value & 35.8 & 34.5 & 37.6 & 27.2 \\
\hline Negative predictive value & 95.0 & 94.1 & 93.8 & 86.9 \\
\hline Tests saved & 42.4 & 42.5 & 37.6 & 34.8 \\
\hline OP missed & 2.9 & 3.4 & 3.9 & 8.6 \\
\hline Cut-off value & 1.3 & 4.5 & -1.0 & 22.6 \\
\hline \multicolumn{5}{|l|}{ Worst any set $B M D \leq-2.5$} \\
\hline Sensitivity & 74.8 & 73.1 & 69.6 & 52.0 \\
\hline Specificity & 70.4 & 69.6 & 75.1 & 71.9 \\
\hline Positive predictive value & 50.1 & 48.8 & 52.5 & 42.3 \\
\hline Negative predictive value & 87.6 & 86.7 & 86.2 & 79.1 \\
\hline Tests saved & 42.4 & 42.5 & 37.6 & 34.8 \\
\hline OP missed & 7.2 & 7.6 & 8.6 & 13.6 \\
\hline Cut-off value & 1.0 & 4.6 & -1.0 & 22.7 \\
\hline
\end{tabular}

Notes: ${ }^{\text {a }}$ Per 100 participants who were candidates for screening BMD and primary prevention. ${ }^{\mathrm{b}} \mathrm{A}$ percentage of participants who had OP according to BMD testing with negative results of predicting tool.

Abbreviations: BMD, body mineral density; BMI, body mass index; FRAX, Fracture Risk Assessment Tool; HF, hip fractures; MOF, major osteoporotic fractures; OSTA, Osteoporosis Self-Assessment Tool for Asians.
Five different diagnostic criteria (WHO, lumbar spine, worst hip, femoral neck, and total hip criteria) were included in the present analysis, and comparisons were made among these different screening tools. It has been reported that the BMD of the femoral neck or total hip might be a better choice than that of the lumbar spine for identifying OP, due to the interference from calcification of the abdominal aorta and osteophytes of the spine at the lumbar spine site. ${ }^{26}$ Some researchers have even suggested that the reference standard should be based on BMD measurement at the femoral neck and total hip. ${ }^{30,31}$ However, considering the low prevalence rates of OP at the femoral neck site (17.2\%) and total hip site $(8.1 \%)$, which did not conform with rates in previous epidemiologic studies, ${ }^{29}$ we applied the WHO criteria $(28.4 \%)$ as the reference diagnostic standard in this study. A low prevalence according to the diagnostic criteria could increase the missed diagnosis of OP. Moreover, the WHO criteria have been most widely approved, and specific bone active treatments, such as antiresorptive or anabolic agents, were all developed on the basis of the WHO criteria. ${ }^{11}$

In the present study, BMI showed limited predictive value for $\mathrm{OP}$ in postmenopausal women compared with the OSTA and FRAX without BMD. Previous investigations indicated that BMI not only performed as a relevant and protective factor for the occurrence and development of $\mathrm{OP}^{24,25,32}$ but also provided a reference for the early diagnosis of OP. In the present study, the AUCs for BMI, ranging from 0.643 to 0.682 , indicated the worst performance for predicting OP among the investigated screening tools according to different criteria. With the WHO criteria, BMI was inferior to the OSTA and FRAX, with an AUC of 0.654 , and the differences were statistically significant $(p<0.05)$. Moreover, BMI also showed a suboptimal sensitivity $(52.0 \%)$ and specificity $(71.9 \%)$ at a cutoff value of $22.7 \mathrm{~kg} / \mathrm{m}^{2}$. When used in screening for OP, BMI was associated with the lowest percentage decrease $(34.8 \%)$ in the subjects for BMD measurement, and also with the highest percentage (13.6\%) of missed OP cases. These results suggested that BMI was not sufficiently effective or reliable for predicting OP in postmenopausal women in Beijing, despite its verified efficacy for predicting OP of elderly Beijing males in our previous study. ${ }^{26}$

Based on age and body weight, the OSTA has been verified in previous studies to be a convenient and valid tool with high sensitivity and acceptable specificity for identifying $\mathrm{OP}$ in postmenopausal women. ${ }^{22,33}$ The 
performance of the OSTA was considered to be better in Chinese women than in men. ${ }^{34}$ According to a BMD T-score reduction of at least -2.5 SDs at different skeletal sites, the AUCs for the OSTA in the prediction of OP indicated good performance and ranged from 0.758 to 0.849 (approximately 0.8 ) in our study, which was consistent with previously reported data. ${ }^{22}$ The optimal cutoff value for the OSTA also varies among different sites of BMD measurement. ${ }^{21,35}$ With the WHO criteria, we confirmed that the optimal cutoff value of -1 provided good sensitivity (69.6\%), high specificity (75.1\%) and negative prediction $(86.2 \%)$. When applied as tool for OP screening, the OSTA was able to reduce the number of cases requiring BMD measurement by $37.6 \%$, at a cost of only missing $8.6 \%$ of OP cases. These results validate the OSTA as a reliable instrument for predicting OP in Han postmenopausal women in Beijing. It was previously reported that the optimal cutoff value and efficacy of this tool may not remain invariable across different areas or countries. ${ }^{34}$ Thus, appropriate adjustment of optimal cutoff values may improve the efficacy of the OSTA, ${ }^{36}$ and revalidating the instrument in accordance with the real conditions among a local population is strongly suggested before using the OSTA for clinical screening for OP. ${ }^{36,37}$

FRAX is well known as a prediction tool for the 10year probability of hip fractures (FRAX-HF) and major osteoporotic fractures (FRAX-MOF), ${ }^{38}$ and it also has been reported to be an effective tool in screening for OP. ${ }^{14,39}$ In our study, the overall AUCs for FRAX without BMD with all diagnostic criteria ranged from 0.728 to 0.855 , performing as well as the OSTA in predicting OP. According to the WHO criteria, the AUC for predicting OP appeared to be greater for FRAX-HF (0.796) than for FRAX-MOF (0.768), and there was significant difference between them $(p<0.05)$. The optimal cutoff values for FRAX-HF and FRAX-MOF were $1 \%$ and $4.6 \%$, respectively, beyond which the tools provided a high sensitivity (74.8\% and $73.1 \%$, respectively) and specificity (70.4\% and $69.6 \%$, respectively). The FRAX-HF and FRAX-MOF could reduce the need for BMD measurement in 42.4$42.5 \%$ of participants with a cost of missing $7.2-7.6 \%$ of the OP patients. In summary, FRAX without BMD was validated to be a reliable screening tool, especially for FRAX-HF. A previous study showed the optimal thresholds of FRAX-HF and FRAX-MOF were $3 \%$ and $6.5 \%$ in Australia, ${ }^{39}$ which indicated that the cutoff value might also be country-specific. FRAX was reported to have limited efficacy in predicting OP for elderly Beijing males in our recent study, ${ }^{26}$ and thus, its predictive value seemed to vary between different genders.

The current study found no statistically significant difference in the AUCs for the OSTA (0.798) and FRAX-HF (0.796) for predicting OP $(p=0.78)$, and thus, the FRAXHF and OSTA yielded reliable and similar assessment abilities for identifying individuals with OP. The simple tool could perform as well as the more complex tool. ${ }^{40,41}$ Hence, the ease of use of the OSTA gives it an advantage over FRAX-HF, ${ }^{40}$ which requires a more complicated calculator and web access, particularly for general medical practitioners in community health service centers or remote areas.

This study was cross-sectional and community-based, not retrospective, and the participants were healthy postmenopausal females. The questionnaire data were collected from communities near Beijing Friendship hospital. BMD measurements were operated by the same-qualified technician. The strict inclusion and exclusion criteria were imposed to exclude the effects of secondary OP, race, nationality, and any anti-osteoporotic medications. All the participants were enrolled consecutively and long-term residents of Beijing. The results offered certain values for both general medical practitioners in community health service centers and physicians in major hospitals for OP screening among the population and reducing the rate of missed diagnosis with omission of BMD measurement.

\section{Limitations}

However, this study also has some limitations. All of the participants were recruited from the communities nearby Beijing Friendship hospital in Xicheng District of Beijing. Thereby, the study population may not completely represent the demographic profiles of the female population in Beijing. Considering the low or high prevalence of OP at different diagnostic criteria in the study, selection bias might be one of affecting factors. A more extensive sampling from multi-communities in different areas of Beijing is expected in the future. Compared with our previous study in elder men, BMI was less predictive in postmenopausal women, the dual effects of BMI should be taken into consideration. Moderate mechanical loading of body weight is beneficial to bone health but obesity is detrimental. Maybe fat mass should be included in further study. Long term follow-up is still needed for assessing the ability of FRAX in predicting 10-year fracture risk. Higher quality researches in randomized design with 
different population-based cohorts are expected in the future. ${ }^{41}$

\section{Conclusions}

The efficacies of three screening tools for predicting OP were evaluated and compared. The results showed that the OSTA and FRAX-HF were qualified and reliable tools for distinguishing OP in Han Beijing postmenopausal women, and both of them were better than BMI. Moreover, the OSTA offers advantages of convenience and simplicity in clinical practice. The optimal thresholds were confirmed, and with these the unnecessary economic cost and radiation exposure from BMD screening could be reduced. We suggest that Han postmenopausal women in Beijing with an OSTA $\leq-1$ and FRAX-HF $\geq 1 \%$ should undergo BMD measurement in the screening of OP.

\section{Acknowledgments}

The study was funded by the Capital Health Project (Z131100006813040), Beijing, People's Republic of China. I would like to show my gratitude to Dr. Fei Qi and Guo Ai who have provided me with valuable guidance to this study. I would also like to thank Wu Xiaoshuang for all her kindness and love.

\section{Disclosure}

The authors report no conflicts of interest in this work.

\section{References}

1. Kanis JA, Cooper C, Rizzoli R, Reginster JY; Scientific Advisory Board of the European Society for Clinical and Economic Aspects of Osteoporosis (ESCEO) and the Committees of Scientific Advisors and National Societies of the International Osteoporosis Foundation (IOF). European guidance for the diagnosis and management of osteoporosis in postmenopausal women. Osteoporos Int. 2019;30(1):3-44.

2. Christiansen C, Peck WA, et al. Consensus development conference: diagnosis, prophylaxis, and treatment of osteoporosis. Am J Med. 1993;94(6):646-650. doi:10.1016/0002-9343(93)90218-E

3. Cummings SR, Melton LJ. Epidemiology and outcomes of osteoporotic fractures. Lancet. 2002;359(9319):1761-1767. doi:10.1016/S01406736(02)08657-9

4. Fujiwara S, Zhao X, Teoh C, Jaffe DH, Taguchi Y. Disease burden of fractures among patients with osteoporosis in Japan: health-related quality of life, work productivity and activity impairment, healthcare resource utilization, and economic costs. J Bone Miner Metab. 2019;37(2):307-318. doi:10.1007/s00774-018-0916-1

5. Qiao D, Liu X, Tu R, et al. Gender-specific prevalence and influencing factors of osteopenia and osteoporosis in Chinese rural population: the Henan Rural Cohort Study. BMJ Open. 2020;10(1):e028593. doi:10.1136/bmjopen-2018-028593

6. Burge R, Dawson-Hughes B, Solomon DH, Wong JB, King A, Tosteson A. Incidence and economic burden of osteoporosis-related fractures in the United States, 2005-2025. J Bone Miner Res. 2007;22 (3):465-475. doi:10.1359/jbmr.061113
7. Liu ZH, Zhao YL, Ding GZ, Zhou Y. Epidemiology of primary osteoporosis in China. Osteoporos Int. 1997;7 Suppl 3:S84-7. doi:10.1007/BF03194349

8. Yang Y, Du F, Ye W, et al. Inpatient cost of treating osteoporotic fractures in mainland China: a descriptive analysis. Clinicoecon Outcomes Res. 2015;7:205-212. doi:10.2147/CEOR.S77175

9. Si L, Winzenberg TM, Jiang Q, Chen M, Palmer AJ. Projection of osteoporosis-related fractures and costs in China: 2010-2050. Osteoporos Int. 2015;26(7):1929-1937. doi:10.1007/s00198-015-3 093-2

10. Kanis JA. Assessment of fracture risk and its application to screening for postmenopausal osteoporosis: synopsis of a WHO report. WHO Study Group. Osteoporos Int. 1994;4(6):368-381. doi:10.1007/ BF01622200

11. Cosman F, de Beur SJ, LeBoff MS, et al. Clinician's guide to prevention and treatment of osteoporosis. Osteoporos Int. 2014;25 (10):2359-2381. doi:10.1007/s00198-014-2794-2

12. Ma Z, Yang Y, Lin J, et al. BFH-OST, a new predictive screening tool for identifying osteoporosis in postmenopausal Han Chinese women. Clin Interv Aging. 2016;11:1051-1059. doi:10.2147/CIA. S107675

13. Kanis JA, Oden A, Johnell O, et al. The use of clinical risk factors enhances the performance of BMD in the prediction of hip and osteoporotic fractures in men and women. Osteoporos Int. 2007;18 (8):1033-1046. doi:10.1007/s00198-007-0343-y

14. Compston J, Bowring C, Cooper A, et al. Diagnosis and management of osteoporosis in postmenopausal women and older men in the UK: National Osteoporosis Guideline Group (NOGG) update 2013. Maturitas. 2013;75(4):392-396. doi:10.1016/j.maturitas.20 13.05.013

15. Kanis JA, Harvey NC, Cooper C, et al. A systematic review of intervention thresholds based on FRAX: a report prepared for the National Osteoporosis Guideline Group and the International Osteoporosis Foundation. Arch Osteoporos. 2016;11(1):25. doi:10.1007/s11657-016-0278-z

16. McCloskey E, Johansson H, Harvey NC, et al. Management of patients with high baseline hip fracture risk by FRAX reduces hip fractures-a post hoc analysis of the SCOOP study. J Bone Miner Res. 2018;33(6):1020-1026. doi:10.1002/jbmr.3411

17. Compston J, Cooper A, Cooper C, et al. UK clinical guideline for the prevention and treatment of osteoporosis. Arch Osteoporos. 2017;12 (1):43. doi:10.1007/s11657-017-0324-5

18. Koh LK, Sedrine WB, Torralba TP, et al. A simple tool to identify asian women at increased risk of osteoporosis. Osteoporos Int. 2001;12(8):699-705. doi:10.1007/s001980170070

19. Chen SJ, Chen YJ, Cheng CH, Hwang HF, Chen CY, Lin MR. Comparisons of different screening tools for identifying fracture/ osteoporosis risk among community-dwelling older people. Medicine. 2016;95(20):e3415. doi:10.1097/MD.0000000000003415

20. Zha XY, Hu Y, Pang XN, Chang GL, Li L. Diagnostic value of osteoporosis self-assessment tool for Asians (OSTA) and quantitative bone ultrasound (QUS) in detecting high-risk populations for osteoporosis among elderly Chinese men. J Bone Miner Metab. 2015;33 (2):230-238. doi:10.1007/s00774-014-0587-5

21. Lu C, Chen D, Cai Y, Wei S. Concordane of OSTA and lumbar spine BMD by DXA in identifying risk of osteoporosis. J Orthop Surg Res. 2006;1:14. doi:10.1186/1749-799X-1-14

22. Yang Y, Wang B, Fei Q, et al. Validation of an osteoporosis selfassessment tool to identify primary osteoporosis and new osteoporotic vertebral fractures in postmenopausal Chinese women in Beijing. BMC Musculoskelet Disord. 2013;14:271. doi:10.1186/1471-2474$14-271$

23. Evans AL, Paggiosi MA, Eastell R, Walsh JS. Bone density, microstructure and strength in obese and normal weight men and women in younger and older adulthood. J Bone Miner Res. 2015;30(5):920928. doi:10.1002/jbmr.2407 
24. Bergkvist D, Hekmat K, Svensson T, Dahlberg L. Obesity in orthopedic patients. Surg Obes Relat Dis. 2009;5(6):670-672. doi:10.1016/ j.soard.2009.05.014

25. Saarelainen J, Kiviniemi V, Kröger H, et al. Body mass index and bone loss among postmenopausal women: the 10-year follow-up of the OSTPRE cohort. J Bone Miner Metab. 2012;30(2):208-216. doi:10.1007/s00774-011-0305-5

26. Zhang X, Lin J, Yang Y, et al. Comparison of three tools for predicting primary osteoporosis in an elderly male population in Beijing: a cross-sectional study. Clin Interv Aging. 2018;13:201-209. doi:10.2147/CIA.S145741

27. Greiner M, Pfeiffer D, Smith RD. Principles and practical application of the receiver-operating characteristic analysis for diagnostic tests. Prev Vet Med. 2000;45(1-2):23-41. doi:10.1016/S0167-5877(00) 00115-X

28. Swets JA. Measuring the accuracy of diagnostic systems. Science. 1988;240(4857):1285-1293. doi:10.1126/science.3287615

29. Cui Z, Meng X, Feng H, et al. Estimation and projection about the standardized prevalence of osteoporosis in mainland China. Arch Osteoporos. 2019;15(1):2. doi:10.1007/s11657-019-0670-6

30. Looker AC, Melton LJ 3rd, Borrud LG, Shepherd JA. Lumbar spine bone mineral density in US adults: demographic patterns and relationship with femur neck skeletal status. Osteoporos Int. 2012;23 (4):1351-1360. doi:10.1007/s00198-011-1693-z

31. Kanis JA, McCloskey EV, Johansson H, Oden A, Melton LJ 3rd, Khaltaev N. A reference standard for the description of osteoporosis. Bone. 2008;42(3):467-475. doi:10.1016/j.bone.2007.11.001

32. Lin J, Ma Z, Fei Q, et al. Association of body mass index and male primary osteoporosis. Zhonghua Yi Xue Za Zhi. 2014;94(33):25932596.

33. Rud B, Hilden J, Hyldstrup L, Hróbjartsson A. The osteoporosis selfassessment tool versus alternative tests for selecting postmenopausal women for bone mineral density assessment: a comparative systematic review of accuracy. Osteoporos Int. 2009;20(4):599-607. doi:10.1007/s00198-008-0713-0
34. Chin KY. A review on the performance of osteoporosis self-assessment tool for Asians in determining osteoporosis and fracture risk. Postgrad Med. 2017;129(7):734-746. doi:10.1080/00325481.2017. 1353394

35. Muslim D, Mohd E, Sallehudin A, Tengku Muzaffar T, Ezane A. Performance of Osteoporosis Self-assessment Tool for Asian (OSTA) for Primary Osteoporosis in Post-menopausal Malay Women. Malays Orthop J. 2012;6(1):35-39. doi:10.5704/MOJ.1203.011

36. Subramaniam S, Chan CY, Soelaiman IN, et al. The performance of osteoporosis self-assessment tool for Asians (OSTA) in identifying the risk of osteoporosis among Malaysian population aged 40 years and above. Arch Osteoporos. 2019;14(1):117. doi:10.1007/s11657019-0666-2

37. Subramaniam S, Ima-Nirwana S, Chin KY. Performance of Osteoporosis Self-Assessment Tool (OST) in predicting osteoporosis-a review. Int $J$ Environ Res Public Health. 2018;15(7):1445. doi:10.3390/ijerph15071445

38. Catalano A, Morabito N, Basile G, et al. Fracture risk assessment in postmenopausal women referred to an Italian center for osteoporosis: a single day experience in Messina. Clin Cases Miner Bone Metab. 2013;10:191-194.

39. Pang WY, Inderjeeth CA. FRAX without bone mineral density versus osteoporosis self-assessment screening tool as predictors of osteoporosis in primary screening of individuals aged 70 and older. $\mathrm{J} \mathrm{Am}$ Geriatr Soc. 2014;62(3):442-446. doi:10.1111/jgs.12696

40. Nayak S, Edwards DL, Saleh AA, Greenspan SL. Systematic review and meta-analysis of the performance of clinical risk assessment instruments for screening for osteoporosis or low bone density. Osteoporos Int. 2015;26(5):1543-1554. doi:10.1007/s00198-0153025-1

41. Rubin KH, Friis-Holmberg T, Hermann AP, Abrahamsen B, Brixen $\mathrm{K}$. Risk assessment tools to identify women with increased risk of osteoporotic fracture: complexity or simplicity? A systematic review. J Bone Miner Res. 2013;28(8):1701-1717. doi:10.1002/jbmr.1956
Clinical Interventions in Aging

\section{Publish your work in this journal}

Clinical Interventions in Aging is an international, peer-reviewed journal focusing on evidence-based reports on the value or lack thereof of treatments intended to prevent or delay the onset of maladaptive correlates of aging in human beings. This journal is indexed on PubMed Central, MedLine, CAS, Scopus and the Elsevier
Bibliographic databases. The manuscript management system is completely online and includes a very quick and fair peer-review system, which is all easy to use. Visit http://www.dovepress.com/ testimonials.php to read real quotes from published authors. 